\title{
XL.-Observations and experiments concerning Mr. Davy's hypothesis of electro-chemical affinity
}

\section{Donovan Esq.}

To cite this article: M. Donovan Esq. (1811) XL.-Observations and experiments concerning Mr. Davy's hypothesis of electro-chemical affinity , Philosophical Magazine Series 1, 37:155, 227-233, DOI: $10.1080 / 14786441108563272$

To link to this article: http://dx.doi.org/10.1080/14786441108563272

Published online: 18 May 2009.

Submit your article to this journal $₫$

Џ Article views: 2

Q View related articles $₫$ 
and obtained a series of irregular polygons, the trace of which indicates the successive intersections of the surface of the soil by horizontal planes raised one metre above each other.

These polygons, more or less distant from each other as the slope of the ground is more or less rapid, indicate to the eye in the most palpable and rigorous manner, the inclination of the hillocks which run parallel with the banks of the Seine, as well as the factitious mounds which we remark in the bottom of the valley, and which we bave al. ready mentioned.

The general survey of the city of Paris was finished in two years. It required operations in detail of a most multifarious kind; and I have no doubt that, by following the order and course which I have pointed out in another memoir *, we shall in a few years be in possession of results equally satisfactory with respect to the general survey of France.

XL.-Olservations and Experiments concerning Mr. Davy's Hypothesis of Electro-chemical Affinity.-By M. DonoVAN, Esq.

Mr. Davy's hypothesis of the identity of chemical and electric attraction, although ably supported by a variety of strong facts, ingenious reasonings, and happy illustrations, is not confirmed by the concordance of known phænomena. Between these powers so many differences are observabie, the one is so often produced where the other can scarcely be supposed to operate, or, according to what is known, ought to operate differently, that we cannot with any degree of confidence rest on the assumption of their similarity; the less so, when it is reflected that the whole is suppurted by probabilities only, without even the profession of a single proof. However truly philosophical may be the design of referring all the operating energies of nature to one great universal cause; yet must we proceed with caution, and not be misled to pronounce causes identical before we are convinced of their real connexion. The limited perceptions of man do not allow him to survey the chain of causes at one steady comprehensive view. There must at length be a break; and when he loses sight of the connexion, he can no longer pronounce upon identity.

The reasonings in the following pages rest not on a hy-

* Journal des Mines, tom. xvii p. 297. 
pothetical assumption of two electric fluids, of one, or of none. In pursuing every investigation, we must reason on doubtful points by the assistance of those that are certainly known on the subject. That of this power, whatever be its nature, there exist two modifications, each of which has a strong tendency to approach the other to an insensible distance, is proved beyond the possibility of doubt; proved by the only means of decision in any matter; namely, the eyidence of the serses. It is proved by no less a certainty, that similar modifications have a tendency to recede from each other; and that where the dissimilar powers are at liberty to come into contact, they effect a mutual change which deprives each of its relative properties. These are all the postulates necessary to the following reasonings; and it is of no consequence what expressions are used, nor whetker positive electricity be considered as plus, and negative as minus ; nor whether both are merely properties of matter, and not the agency of any fluid sui generis. The facts are indisputable, notwithstanding the insufficiency of language. But as the language of the hypothesis which supposes the existence of two fluids is most easy of exnression, it shall be hereafter adoptcd.

Mr. Davy has stated bis opinon, that the effects altributed to a certain force called affinity, exerting its energy hetween the ultimate particles of heterogeneous matter, may be no more than the operations of electricity; and thinks that all the phænomena of affinity ought to happen conformably to the known principles of electricity.

He supposes that comitined bodies are in differently electrical states: that acids and oxygen are in the negative state; that earths, alkalies, wetals, oxides and inflammables are in the positive state: that the attraction of these ditferent powers is the force which sustains these substances in combination.

He illustrates such a combination by the example of Beccaria's glass plates, differently electrified, which cohere with great force; and adds, that "different particles in combining must be still supposed to preserve their peculiar states of cnergy."

He instances a variety of substances, as metals, acids and alkalies, which by a few contacts become electrical in different states.

Mr. Davy lastly supposes that artificial electricity effects the decomposition of those compounds, by attracting the different and by repelling the similar power.

Mr. Davy, it is plain, sets out with two assumptions. 
Mr. Davy's Hypothesis of Electro-chemical Affinity. 820

1st. That bodies which possess for each other a chemical affinity are in different states of electricity.

2d. That these bodics after combination still retain their different electricities.

These Mr. Davy has not proved. Were they allowed, he certainly succeeds in establishing some of his points. But are we to grant for that reason what is entirely in opposition to the laws of electricity, which have been founded un the firm basis of experiment?

In opposition to these two assumptions, I shall endeavour to show:

1st. That permanently supernatural states of electricity cannot exist.

2d. That different states, after union, no longer display sensible properties.

Entering on the inquiry, the question naturally occurs, How do these substances, when about to combine, acquire differently electrical states? Whether do bodies possess an independent state of power, absolutely existing; or is it always acquired in consequence of separation afier contact? The former can scarcely be allowed, from the following considerations :

1st. Electricity can only exist in three states : in the naural, in the positive, or in the negative state.

2d. The first is the state in which electricity always insensibly exists, until some action te exerted on it which causes it to become sensible, and then it appears in either the positive or negative state.

3d. The positive and negative are forced states, which can only exist while the primary exciting cause continues, unless they be detained by substances which allow of but a very slow passage. Even then, so transitory is their existence, that they will insensibly disappear, once more forming natural electricity.

These are facts that cannot be controverted. Sulplur, wax, glass, resin, silk, \&c., when excited either strongly or feebly, will always in a little time return to their natural state. The stronger electricities of charged conductors and the condensed powers of batteries soon undergo the same change. Thus we are acquainted with no substance which has the property of retaining a forced state permanently.

From observation of the fact, that some bodies when brought into contact and afterwards separated show signs of being differently electrified, it might appear that the attraction between such bodies would be a sufficient cause of 
combination. For at every effort towards separation the attraction begins to act, which apparently would permanently unite them.

Mr. Davy has no where expressly mentioned his opinion that electric energies may be produced in this manner. Yet that he has it in view, I think, is deducible from his illustrations of the electricities evolved by separation after contact, and of the energies being often increased by heating; all of which are apparent after separation only.

The improbability of this opinion becomes apparent; when it is considered, that although bodies are rendered electrical by separation, yet there are no grounds for supposing electricity evolved by contact. On the contrary, it is certain that forced states cannot exist together for any considerable length of time, especially if the contiguous bodies be metallic: and accordingly such bodies, while the contact continues, display no powers. Even by the hypothesis, electricity is rendered sensible by separation only. Now as it will readily be granted that a power cannot act before it is created, I would ask, What is the cause of the combination of solids, which are attended by a complete change of properties, by a violent evolution of heat and light, and other marked effects; which solids, fronk their state of existence, are sufficiently protected by aggregation from efforts towards separation by any mechanical cause?

Since bodies which possess an affinity cannot exist in independently electric states, and since electricity evolved. by separation is shown to be a cause insufficient for combination, I see no foundation for Mr. Davy's first assumption, - - that heterogenous particles of matterare in differently: electrical states; but consider it as irreconcileable to the established laws of electricity and to reason.

The second assumption, - that different particles of matter in combining must still be supposed to retain their peculiar states of energy,--comes next under consideration.

I begin by stating the ptincipal evidence in favour of this supposition. When a compound is decomposed by Voltaic electricity, each component is invariably attracted to a determinate pole. The question stands fairly thus: "Why is the one substance always attracted to a determinate pole, suppose to the negative? Because it is naturally positive. But how is it proved that it is naturally positive? Because it is attracted to the negative pole." There is no service rendered to the hypothesis by this circular reasoning.

I shall now submit the evidence against this supposition. 
If from the ends of two equally and differently electrified conductors be suspended two gilt pith balls, by gilt strings, the balls will attract each other; but when they come into contact, a mutual annihilation of power takes place; the balls separate, and no longer show signs of electricity.

Or a metallic sphere on which is erected a quadrant electrometer may be insulated and positively electrified, the pith ball rises: if some negative sparks be thrown on, the ball-sinks. Were another example necessary of so well known a fact, I would instance two jars differently electrified. Each of them separately produces the most violent effects. It will ignite, fuse and burn the most refractory metals. It will pour out torrents of fire on contiguous bodies, it will instantly deprive animals of life. When a proper comununication with the other jar, which is equally charged, is formed, an explosion follows; and instead of either jar being possessed of the additional charge of the other, they are both found harmless and altogether deprived of electricity. All these instances demonstrate in the clearest manner the destruction of forced states, when they come into contact.

As to Mr. Davy's example of Beccaria's plates, it is plain that the cobesion depends entirely on the nonconducting power of the glass; for the opposite powers are thus prevented from coming quickly into contact. The annihilation is effected in some time, and the plates separate. It will be shown, however, that combined particles are in circumstances very different from Beccaria's plates.

If it be proved that matter after combination cannot retain a forced state, the doctrine of combination itself falls to the ground. For bodies in solution which possess affinity should, in the manner of other bodies differently electrified, first combine; and afterwards, obeying any tendency towards separation, as difference of specific gravity, they should separate as goon as the annihilation took place.

It might be imagined that when the bodies are in the fluid state, a separation and consequent evolution of new powers might take place, which would still maintain the compound.

It were unphilosophical to attribute unnecessarily the same natural effects to dissimilar causes. Combination, whether between fluids or solids, may reasonably be pronouncer the operation of the same power. There is, however, a stronger objection. The particles of combining substances are surrounded by a conducting medium (water). The moment that different electricities would be produced, $P_{4}$ 
they would be annihilated by the intervention of the fluid.

The objection might offer, that water is not a conductor of low intensities, for instance of the Voltaic. But this does not affeet the force of the above reasoning. For, whatever be the intensity supposed to maintain chemical combinations, it is certainly conducted by water; as is proved by immersing after separation any of these substances which become electric by contact, in water. They lose all signs of electricity, and appear in the natural state.

There are other instances wherein the medium is a conductor, and consequently admits not of differently co-existing electricities. All electrics at a high temperature become conductors. Yet many chemical combinations take place at a high temperature only. But how much is this difficulty increased when applied to metallic alloys! the high temperature of which in forming should considerably assist their natural conducting powers.

I scarcely imagine that any one would instance the zones of different electricities producible in a long conductor electrified by a weak power. It nust be recollected that with regard to metallic alloys there can be no zones, the whole mass being composed of pairs of atoms united, as it is said, by electric attraction.

In the same manner, so little capable of retaining different electricities is a vacuum which is also a conductor, that, as Beccaria and others have shown, the transmission and annibilation of powers take place insensibly from pith balls, without a change of place in these bodies.

These nbservations are applicable, whether we consider difference of powers as owing to independent states essential to different bodies, or as always evolved by separation after contact.

The tendency of the preceding observations was to show that Mr. Davy's two assumptions are not only without proof, but entirely in opposition to any thing we know of electricity. I shall now endeavour to show, that even allowing combining bodies to be in different states, and that in uniting they do preserve their peculiar energies, yet still that these powers are inadequate to account for the phænomena attributed to affinity.

If there exist no such power as that which has been called affinity, and if all the phænomena of combination be caused by the differently electrical states of bodies, it stould follow that,

1. To produce combination, it is only necessary ta present 
sent to each other the combining bodies in opposite states of electricity.

2. To counteract combination; or, if formed, to subvert it : all that is necessary is to induce a similar state of electricity in the bodies; or, which is the same thing, to give one a state contrary to that which it naturally possesses.

The latter of these positions Mr. Davy says he has proved by experiment.

In contradiction to these consequences deduced from the hypothesis, I shall produce instances in which,

1. Bodies in opposite states of electricity shall not combine when present to each other.

2. Bodies in similar states shall combine with as much force as if in different states.

And I shall show that Mr. Davy's experiment does not prove its object.

Were the former position of the above consequences true, we should be in possession of an easy method of forming combinations at present believed to be impracticable, as in the same manner substances have been decompounded which never yielded to other means. Thus we should produce all the salts formed by silica with acids; we should unite gold and platina with nitric acid: nay, if there be not that specific attraction attributable to the ultimate particles of heterogeneous matter only, we should combine iron with iron, or potash with potash: and these similar substances held together by different states of electricity should, when placed in the Voltaic circuit, arrange themselves, of,serving the law of other compounds, according to their respective attractions.

The well established law, that affinity exerts its influence at insensible distances only, ought not to be true; since electricity to exert its attraction does not require an insensible distance. On this account we should also produce all compounds by a mixture of its dry ingredients in fine powder; at least when forced to approach by strong pressure.

[To be continued]

XLI. Proceedings of Learned Societies.

ROYAL SOCIETY.

March 1st and 7 th. $-T_{\text {HE }}$ President in the chair. A paper by Mr. Knight was read, detailing experiments on the manner in which plants shoot forth their radicles. In a former 